\title{
Erratum to: Houttuynia cordata aqueous extract attenuated glycative and oxidative stress in heart and kidney of diabetic mice
}

\author{
Cheng-chin Hsu ${ }^{1,5} \cdot$ Hui-ting Yang ${ }^{2} \cdot$ Jing-jing $\mathrm{Ho}^{2} \cdot$ Mei-chin Yin ${ }^{2,3} \cdot \mathrm{Jen}^{-y i n g} \mathrm{Hsu}^{4}$
}

Published online: 22 April 2017

(C) Springer-Verlag Berlin Heidelberg 2017

\section{Erratum to: Eur J Nutr (2016) 55:845-854 DOI 10.1007/s00394-015-0994-y}

In original article, few western blot bands of p38, JNK, p-JNK, ERK1/2 or GAPDH in Fig. 3 are questioned to be similar and confused. Although these original data are not wrong, authors would like to use the following western blot bands as replacements in order to avoid confusion.

These replacements do not change the results and conclusions of this original article.

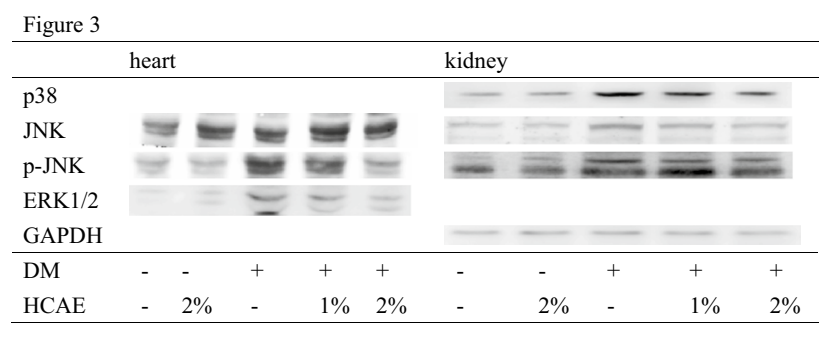

The online version of the original article can be found under doi:10.1007/s00394-015-0994-y.

\section{Mei-chin Yin}

mcyin@mail.cmu.edu.tw

1 School of Nutrition, Chung Shan Medical University, Taichung City, Taiwan

2 Department of Nutrition, China Medical University, 91, Hsueh-shih Rd., Taichung City, Taiwan

3 Department of Health and Nutrition Biotechnology, Asia University, Taichung City, Taiwan

4 School of Pharmacy, China Medical University, Taichung City, Taiwan

5 Department of Nutrition, Chung Shan Medical University Hospital, Taichung City, Taiwan 